\title{
Near-surface microstructure of Pt-Rh(110) and (111) surfaces
}

\author{
Ch. Steiner, ${ }^{1}$ B. Schönfeld, ${ }^{1}$ M. M. I. P. van der Klis, ${ }^{1}$ G. Kostorz, ${ }^{1}$ B. D. Patterson, ${ }^{2}$ and P. R. Willmott ${ }^{2}$ \\ ${ }^{1}$ ETH Zürich, Institute of Applied Physics, CH-8093 Zürich, Switzerland \\ ${ }^{2}$ Paul Scherrer Institut, Swiss Light Source, CH-5232 Villigen PSI, Switzerland \\ (Received 26 October 2005; revised manuscript received 25 January 2006; published 9 May 2006)
}

\begin{abstract}
Diffuse $\mathrm{x}$-ray scattering under grazing incidence was measured of Pt-47 at. \% $\mathrm{Rh}(110)$ and (111) surfaces at $1000 \mathrm{~K}$. The elastic scattering contributions of short-range order scattering and displacement scattering were separated from the inelastic scattering contributions that served for data calibration. The modulations in shortrange order scattering are small and indicate differences in the near-surface microstructure of the two surfaces: a global maximum at $1 \frac{1}{2} 0$ (as for the bulk microstructure) for the (110) surface and at $\frac{1}{2} \frac{1}{2} \frac{1}{2}$ positions for the (111) surface. The dominant nearest-neighbor configuration of both surfaces is reminiscent of the segregation profile of a (111) surface. A tendency towards the bulk "40" structure is only noted for the (110) surface.
\end{abstract}

DOI: 10.1103/PhysRevB.73.174205

PACS number(s): 61.66.Dk, 68.55.Jk

\section{INTRODUCTION}

The bulk microstructure of single-crystalline Pt- 47 at. \% $\mathrm{Rh}$ quenched from $923 \mathrm{~K}$ was recently studied by applying wide-angle diffuse $\mathrm{x}$-ray scattering and small-angle neutron scattering. ${ }^{1}$ In contrast to current phase diagrams that give a broad miscibility gap below about $1030 \mathrm{~K},{ }^{2}$ local order was revealed. It was characterized by small deviations of the short-range order scattering from the monotonic Laue scattering, with a maximum at $1 \frac{1}{2} 0$. Linear displacement scattering was easily recognized already in the raw data although the atomic sizes of the constituents only differ by $3 \%$.

Because of the interest in technical applications of Pt-Rh as a catalyst as well as for basic research, Pt-Rh surfaces have frequently been investigated using surface-sensitive techniques. Early atom-probe field-ion microscopy (APFIM) investigations of (100) and (111) surfaces of alloys with about 50 at. \% Rh revealed that Pt is enriched in the top layer. ${ }^{3,4}$ Oscillatory segregation profiles comprising a few layers were resolved. ${ }^{5}$ If sulphur, even at a low level of only $50 \mathrm{ppm}$, is present as impurity, the top layer will be Rh enriched and the second layer Pt enriched. ${ }^{3}$ Model calculations ${ }^{6-8}$ confirmed that the top surface layer of $\mathrm{Pt}-\mathrm{Rh}$ is $\mathrm{Pt}$ enriched, and details of the segregation profiles were reproduced in these theoretical results.

Differences were noted in the segregation profiles of (100), (110), and (111) surfaces. ${ }^{5,9-12}$ For a clean (100) surface (free of carbon) of Pt-75 at. \% Rh, no oscillatory profile was observed by low-energy electron diffraction (LEED), but a Pt depletion in several layers below the Pt-enriched top layer. ${ }^{9}$ The possibilities of a different composition (75 at. \% $\mathrm{Rh}$ instead of 45 at. \% Rh), a higher annealing temperature (1425 K instead of $975 \mathrm{~K}$ ), or a difference in carbon impurity were discussed to account for the fact that an oscillatory segregation profile is no longer found, in contrast to results from previous AP-FIM investigations. ${ }^{5}$

For Pt-75 at. \% $\mathrm{Rh}(111)$, an oscillatory segregation profile was confirmed by LEED. ${ }^{9}$ For Pt-50 at. \% Rh(110), an oscillatory segregation profile was resolved for the top five layers by LEED..$^{10}$ The (110) surface is reconstructed; there is a $(1 \times 2)$ missing-row reconstruction for Pt-75 at. \% Rh and a
$(1 \times 3)$ missing-row reconstruction for Pt-50 at. \% Rh. ${ }^{10,11}$ Using low-energy ion scattering (LEIS) at elevated temperatures, no $(1 \times 2)$ missing-row reconstruction was any longer found above about $1120 \mathrm{~K} .^{12}$

Kinetics for achieving the equilibrium segregation profile in Pt-Rh were followed at elevated temperatures by LEIS (Refs. 9 and 12) and, following different aging treatments, for quenched samples by AP-FIM, ${ }^{4}$ LEED, Auger-electron spectroscopy (AES), and scanning tunneling microscopy (STM). AP-FIM showed a decrease of the amplitude of the oscillations with increasing temperature [for the (001) surface between 975 and $1175 \mathrm{~K}] .{ }^{4}$ LEIS indicated a reversible change in composition of the top layer of Pt-75 at. \% $\mathrm{Rh}(111)$ between 575 and $1375 \mathrm{~K}$, but accompanying AES investigations of quenched samples indicated that for the deeper layers no equilibrium is reached below $1375 \mathrm{~K}$ within 5 min. ${ }^{9}$

Using STM with chemical contrast, up to three shells of the pair correlation functions in the top layer were determined for Pt-75 at. \% Rh(100), (110), (111) and aging temperatures between 975 and $1425 \mathrm{~K} .{ }^{11}$ Local order was found for the (111) surface, with values of the autocorrelation between $-0.044(13)$ and $-0.069(9)$ for the nearest neighbors, without any systematic temperature dependence. For the (110) surface after aging at elevated temperature, local order was also observed. For the (100) surface, local decomposition was found at $875 \mathrm{~K}$ and local order at $1175 \mathrm{~K}$, the magnitude of the nearest-neighbor autocorrelation being much larger than for the other two surfaces (up to 0.17).

The near-surface microstructure, the microstructure between surface and bulk, has not been characterized yet. This is the domain of $\mathrm{x}$-ray diffuse scattering under grazing incidence; ${ }^{13,14}$ by tuning the angle of the incoming $\mathrm{x}$ rays below the critical angle for total reflection, about the top 10 atomic layers may be investigated. A straightfoward approach to determine the near-surface pair correlation function was employed; only in-plane scattering was taken and a global analysis, based on the assumption of homogeneous local order and cubic symmetry in this region, was applied. For a direct comparison with the previous bulk investigations, ${ }^{1}$ the same single-crystalline Pt-47 at. \% Rh alloy 
was used and a comparable elevated temperature was chosen. In addition, a possible dependence of the microstructure on the type of surface was addressed by investigating the high-symmetry surfaces, (110) and (111), under UHV conditions.

\section{THEORY}

The elastic coherent diffuse scattering $I_{\text {diff }}$ from crystalline solid solutions is due to the presence of different types of atoms that are not strictly periodically arranged on a lattice (short-range order scattering $I_{\mathrm{SRO}}$ ) and to the static local deviations of the atoms from the sites of the average lattice (displacement scattering approximated by size effect scattering $I_{\mathrm{SE}}$ and Huang scattering $I_{\mathrm{H}}$ ). ${ }^{15-17}$ For a cubic binary $A-B$ alloy the diffuse scattering intensity is given by

$$
I_{\mathrm{diff}}(\mathbf{h}) / N=c_{A} c_{B}\left|f_{A}-f_{B}\right|^{2}\left[I_{\mathrm{SRO}}(\mathbf{h})+I_{\mathrm{SE}}(\mathbf{h})+I_{\mathrm{H}}(\mathbf{h})\right],
$$

with

$$
I_{\mathrm{SRO}}(\mathbf{h})=\sum_{l m n} \alpha_{l m n} \cos \left(\pi h_{1} l\right) \cos \left(\pi h_{2} m\right) \cos \left(\pi h_{3} n\right),
$$

where $N$ is the total number of atoms in the crystal, $c_{\mu}$ is the atomic fraction of the component $\mu=A, B, f_{\mu}$ its atomic scattering factor, $\mathbf{h}=\left(h_{1}, h_{2}, h_{3}\right)$ is the scattering vector in reciprocal lattice units $2 \pi / a$ (r.l.u., $a=$ lattice parameter), and the $\alpha_{l m n}$ are the Warren-Cowley short-range order parameters ${ }^{18}$ for the $l m n$ type of neighbors $(l, m, n$ in units of $a / 2)$. The prefactor in Eq. (1), $c_{A} c_{B}\left|f_{A}-f_{B}\right|^{2}$, is called one Laue unit (L.u.). For a statistically uncorrelated arrangement of atoms on a lattice, all $\alpha_{l m n}$ are zero, except for $\alpha_{000}$ which is unity, and short-range order scattering simplifies to the monotonic Laue scattering.

The leading term in displacement scattering, size-effect scattering, is given by

$$
I_{\mathrm{SE}}(\mathbf{h})=\sum_{i} h_{i} Q_{i}(\mathbf{h})
$$

with, e.g.,

$$
Q_{x}(\mathbf{h})=\sum_{l m n} \gamma_{l m n}^{x} \sin \left(\pi h_{1} l\right) \cos \left(\pi h_{2} m\right) \cos \left(\pi h_{3} n\right),
$$

where the coefficients $\gamma_{l m n}^{x}$ represent linear combinations of the species-dependent static atomic displacements.

To separate the various scattering contributions, the leading Fourier coefficients were least-squares fitted, an appropriate procedure if scattering is weakly modulated. In addition, as the number of symmetry-equivalent positions is small because of the softness of the radiation used in grazing incidence, the separation techniques of Borie-Sparks ${ }^{19}$ or Georgopoulos-Cohen ${ }^{20}$ are not suitable. Finally, a restricted number of Fourier coefficients may only be recovered and no species-dependent displacements were considered, as the scattering intensities were restricted to positions in single planes. Sets of three-dimensional Fourier coefficients were fitted as the near-surface microstructure is three dimensional.

\section{EXPERIMENT}

For scattering under grazing incidence, flat sample surfaces are required, with the surface roughness as small as possible. In this work, slices of Pt-Rh with a [110] or a [111] surface normal were cut by spark erosion from a Pt47 at. \% Rh single crystal that had been used previously for bulk measurements. ${ }^{1}$ The sample disks had an oval shape with a diameter between 10 and $12 \mathrm{~mm}$ and a thickness of $3 \mathrm{~mm}$. They were mechanically lapped and polished on a Logitech PM5 lapping machine. Final lapping was done with a $\mathrm{SiO}_{2}$ suspension (grain size of $0.04 \mu \mathrm{m}$ ). The misorientation between the surface normal and the nominal crystallographic direction of the disks was checked optically and with $\mathrm{x}$ rays. It was less than $0.05^{\circ}$ for the region used for the measurements under grazing incidence.

The samples were mounted in a portable UHV chamber (base pressure $<10^{-10} \mathrm{mbar}$ ). They were repeatedly sputtered with $1.5 \mathrm{keV}$ Ar ions at $770 \mathrm{~K}$ for $10 \mathrm{~min}$ and annealed at $1170 \mathrm{~K}$ for $1 \mathrm{~h}$. After the final sputtering, the samples were annealed at $1070 \mathrm{~K}$ for several days. The temperature of the samples during the measurements was $1000 \mathrm{~K}$. The mosaicity of the near-surface microstructure was about $0.05^{\circ}$ full width at half-maximum (in comparison, the bulk mosaicity was larger by a factor of 3 to 4 ).

The UHV chamber has a cylindrically shaped Be window, providing a nearly $360^{\circ}$ access around the sample surface normal. The chamber was mounted on a hexapod which is part of the $(2+3)$ surface diffractometer at the Materials Science Beamline $4 \mathrm{~S}$ at the Swiss Light Source (PSI Villigen, Switzerland). ${ }^{21}$ The horizontal as well as the vertical setup of the hexapod were employed to benefit from the better vertical focusing and the horizontal polarization of the incoming $\mathrm{x}$ rays, respectively. $\mathrm{X}$ rays with an energy of $9.659 \mathrm{keV}$ were used. With the values $\delta=2.98(5) \times 10^{-5}$ and $\beta$ $=2.00(5) \times 10^{-6}$ for the index of refraction $n=1-\delta-i \beta$ (the data for anomalous scattering were taken from Sasaki ${ }^{22}$ ), a critical angle $\alpha_{c}$ of $0.45^{\circ}$ is obtained.

The incoming beam was focused on the sample, the divergence of the outgoing beam was defined by slits to $\pm 0.05^{\circ}$. The surface region investigated was about $1 \mathrm{~mm}$ in width for the incoming beam and $3 \mathrm{~mm}$ for the outgoing beam. The angle of incidence was $0.33^{\circ}$ for the (110) surface and $0.4^{\circ}$ for the (111) surface, for the outgoing angle the value of incidence was chosen.

Scattering intensities were taken with a Cyberstar detector. About 700 positions for the (110) surface and 550 positions for the (111) surface were measured on a mesh of 0.1 r.l.u. Typical intensities were 400 to 1000 counts per $200 \mathrm{~s}$ for the (110) surface and 1500 to 2500 counts per $200 \mathrm{~s}$ for the (111) surface. The scattering vector was in the range of $0.15-3.5$ r.l.u. for the (110) surface and $0.28-2.8$ r.l.u. for the (111) surface.

Background (electronic noise and sample environment) was registered for both planes on a coarser grid of 0.2 r.l.u. In the measurement of the (110) surface, the background was nearly constant and amounted to 40 counts $/ 200 \mathrm{~s}$, in the measurement of the (111) surface, it was higher and modulated (400-800 counts/200 s) due to a different alignment of the beam optics. 


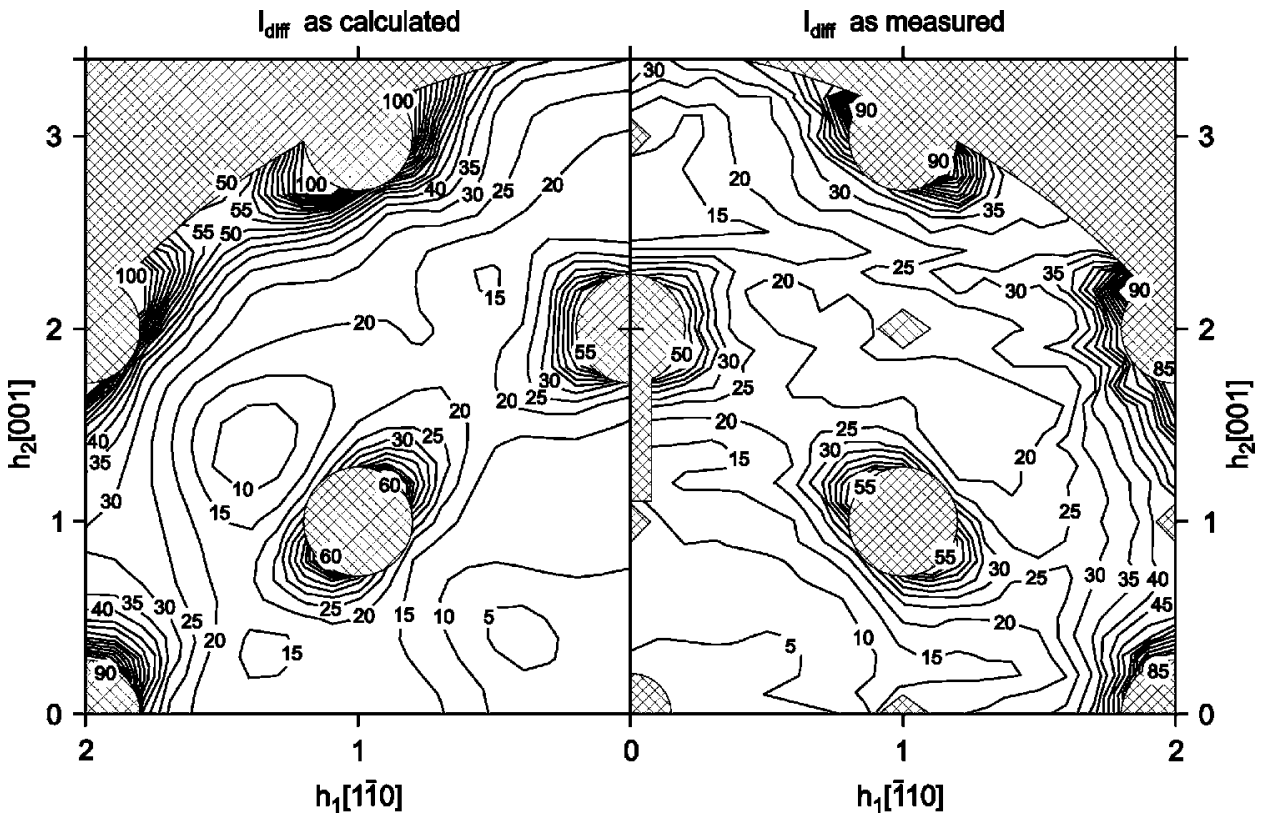

FIG. 1. Calculated (left-hand side) and measured (right-hand side) elastic and inelastic scattering intensity of the Pt-Rh(110) surface, in 0.1 Laue units.

\section{DATA TREATMENT}

Data were corrected for background and the variation of the active sample area $C_{\text {area }}$ with changing scattering vector ${ }^{23}$ and treated within the distorted-wave Born approximation ${ }^{24}$

$$
I_{\text {corr }}(\mathbf{h})=\frac{I(\mathbf{h})}{C_{\text {area }} p\left|t_{\text {in }}(\mathbf{h})\right|^{2}\left|t_{\text {out }}(\mathbf{h})\right|^{2}} .
$$

For the calculation of the polarization factor $p$, complete polarization in the horizontal plane was assumed for the incoming $x$ rays. The transmission factors $t_{\text {in }}(\mathbf{h})$ and $t_{\text {out }}(\mathbf{h})$ of the incoming and the outgoing beam differed because of the slight surface misorientation.

For the calculation of thermal diffuse scattering up to third order, bulk elastic constants $c_{i j}$ were employed. ${ }^{25}$ The elastic constants are not known experimentally for $1000 \mathrm{~K}$. Based on their values at room temperature ${ }^{1}$ (with standard deviations of less than $1 \%$ ) and the temperature dependence of the $c_{i j}$ for both elements, ${ }^{26,27}$ the values of $c_{11}=321 \mathrm{GPa}$, $c_{12}=218 \mathrm{GPa}$, and $c_{44}=111 \mathrm{GPa}$ were estimated.

The dynamic Debye-Waller factor may be determined on the basis of these elastic constants. Together with the static Debye-Waller factor (using the bulk lattice parameter change with temperature), a value of $B=0.63 \times 10^{-2} \mathrm{~nm}^{2}$ is obtained for the total (static and dynamic) Debye-Waller factor $\exp \left[-2 B(h / 2 a)^{2}\right]$. However, a much more rapid decrease in Bragg intensities with scattering vector was found for the (111) surface. As a rapid decrease in Bragg intensities was also noted at room temperature, the larger value of $B$ may be caused by a larger static Debye-Waller factor. Because of the small number of Bragg reflections accessible for the given wavelength, it was not possible to determine the total DebyeWaller factor experimentally with sufficient precision. In the following, the value of $B$ was taken as a fitting parameter. It was considered best when the separated short-range order scattering gave the theoretical value of 1 Laue unit. The values of $B$ thus determined were $0.5(1) \times 10^{-2} \mathrm{~nm}^{2}$ for the
(110) surface and $6.5(5) \times 10^{-2} \mathrm{~nm}^{2}$ for the (111) surface.

The scattering intensities were calibrated by comparing the intensities within 0.25 r.l.u. around the Bragg reflections with those of the calculated thermal diffuse scattering and Compton scattering. ${ }^{28}$ This region is strongly dominated by thermal diffuse scattering and thus suited for an approximate calibration unless the alloy is locally decomposed. For a fixed value of $B$, the calibration factor was set such that this ratio became 1 . With a value of $\alpha_{000}$ close to one (within standard deviations) in the subsequent least-squares fitting, the value of $B$ was considered reasonable. Varying the value of $B$ within $5 \%$ leads to a change of all Fourier coefficients (including $\alpha_{000}$ ) within two standard deviations.

\section{RESULTS}

The measured diffuse scattering in 0.1 Laue units of the (110) and the (111) surfaces is shown in Figs. 1 and 2. The hatched areas mark the regions around the Bragg reflections, the direct beam and the crystal truncation rods that were not considered in evaluating the elastic diffuse scattering. The most prominent intensity modulation arises towards the Bragg reflections because of thermal diffuse scattering. The calculated thermal diffuse scattering shown in Fig. 3 for the (110) and (111) surfaces, amounts to $60 \%$ and $40 \%$, respectively, of the total scattering intensity in the region off the Bragg reflections.

No increase in scattering towards the direct beam is visible for both surfaces as close as 0.15 r.l.u., with the intensity being less than 1 L.u. Thus, there is no indication for local decomposition. For the (111) surface, a diffuse maximum is seen at about $\overline{0.7} \overline{0.7} 1.4$ positions. This is no Lifshitz position for an fcc lattice, it is close to a 111 position and mainly due to thermal diffuse scattering. No diffuse maximum is seen for the (110) surface.

With respect to linear displacement scattering, an asymmetry in scattering across Bragg positions is barely 


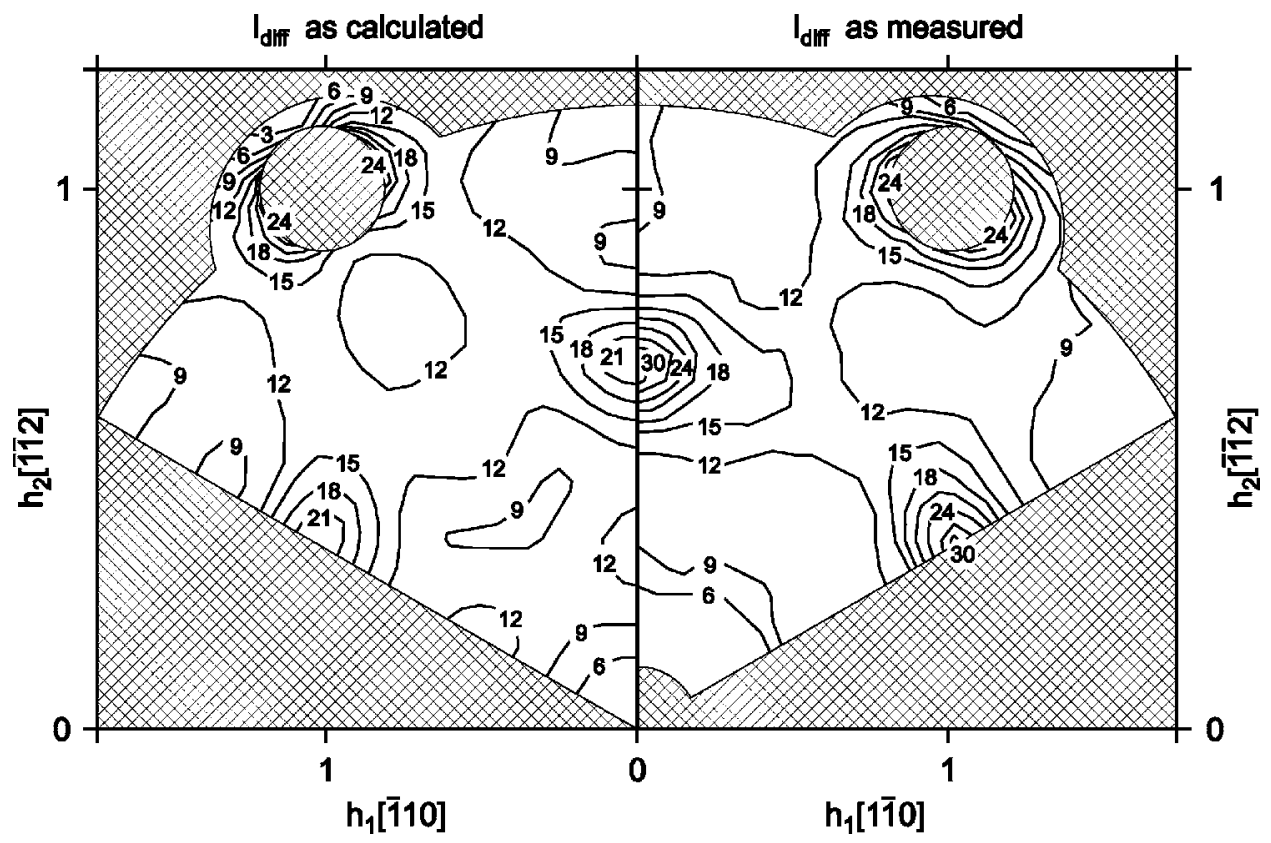

FIG. 2. Calculated (left-hand side) and measured (right-hand side) elastic and inelastic scattering intensity of the Pt-Rh(111) surface, in 0.1 Laue units.

visible-at best for $0 \overline{2} 2$ in Fig. 2. Measurements of the bulk microstructure, on the other side, showed a strong asymmetry across the 220 reflection (see Fig. 3 in Ref. 1).

The number of fitted Warren-Cowley short-range order parameters $\alpha_{l m n}$ and linear displacement parameters $\gamma_{l m n}^{x}$ was chosen such that the $R$ value in the least-squares fitting no longer decreased significantly with an increasing number of Fourier coefficients and the standard deviations were still typically smaller than the magnitude of the fitted parameters. To consider uncertainties in the thermal diffuse scattering, one parameter of the quadratic displacement scattering, i.e., $\delta_{000}^{x}$, was taken into account. This parameter also considers quadratic elastic displacement scattering (Huang scattering) to lowest order. Its value is small, $-0.05(1)$ for the (110) surface and $-0.08(1)$ for the (111) surface, and mainly af- fects the value of the short-range order parameter $\alpha_{000}$. With 10 parameters altogether, the number of fitted Fourier coefficients is small. The Fourier coefficients are listed in Tables I and II.

\section{A. Short-range order scattering}

The nearest-neighbor Warren-Cowley short-range order parameter $\alpha_{110}$ is negative for both surfaces, indicating local order. Also, the magnitude is small as for the bulk microstructure. ${ }^{1}$ In Fig. 4 the recalculated short-range order scattering $I_{\text {SRO }}$ is shown in 0.1 Laue units for a (001) plane using the data of both surfaces (Table I). The intensities are weakly modulated, but clearly differ for both surfaces. For the (110) surface, the maximum of 1.5 L.u. is located at $1 \frac{1}{2} 0$

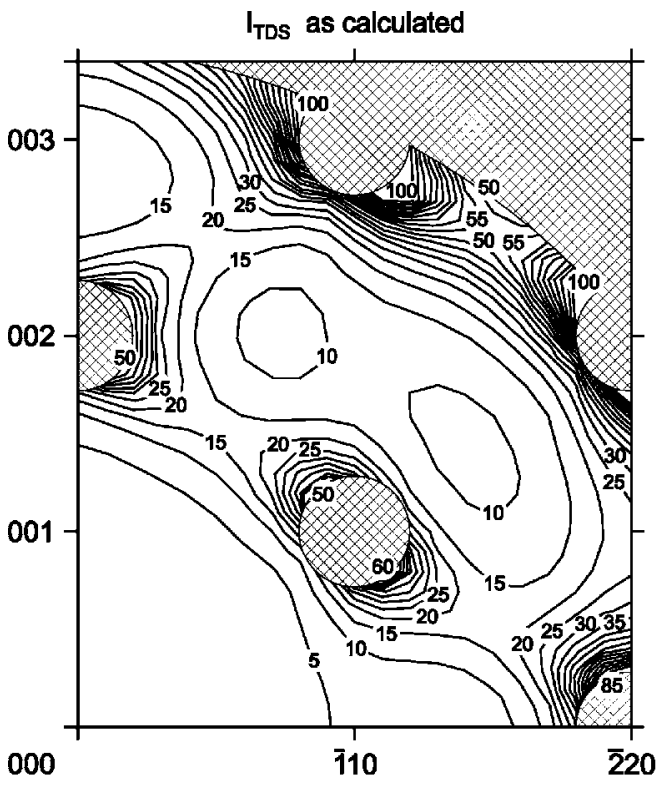

(a)

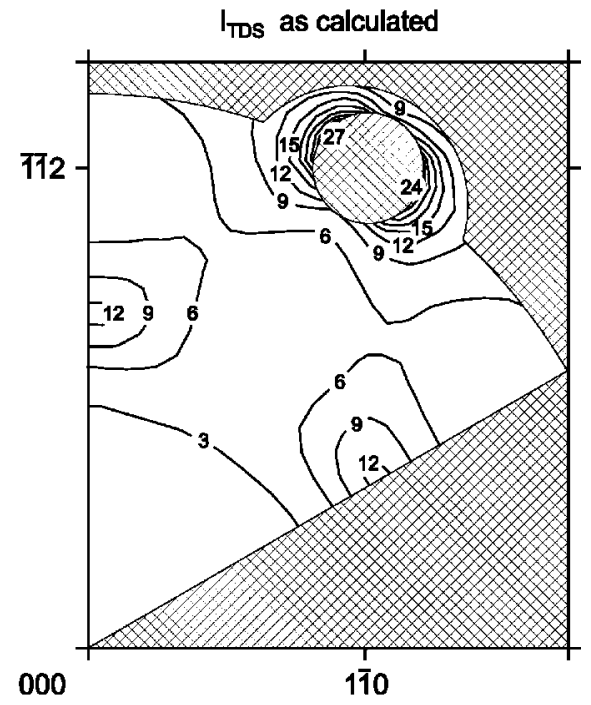

(b)
FIG. 3. Calculated thermal diffuse scattering $I_{\mathrm{TDS}}$ of (a) the Pt$\mathrm{Rh}(110)$ and (b) the Pt-Rh(111) surface in 0.1 Laue units. 
TABLE I. Fitted Warren-Cowley short-range order parameters $\alpha_{l m n}$ for the Pt-47 at. \% Rh(110) and (111) surface at $1000 \mathrm{~K}$.

\begin{tabular}{ccc}
\hline \hline & & $\alpha_{l m n}$ \\
$l m n$ & $(110)$ surface & $(111)$ surface \\
\hline 000 & $0.927(57)$ & $0.926(30)$ \\
110 & $-0.056(8)$ & $-0.023(5)$ \\
200 & $0.057(10)$ & $-0.077(9)$ \\
211 & $0.014(6)$ & $-0.003(4)$ \\
220 & $-0.022(7)$ & $0.032(6)$ \\
310 & & $-0.013(4)$ \\
\hline \hline
\end{tabular}

positions, it is also the global maximum. The intensity distribution resembles the pattern found in bulk Pt-Rh. ${ }^{1}$ For the (111) surface, the recalculated scattering has the highest intensity of 1.1 L.u. at 100 , the global maximum with 1.7 L.u. is located at $\frac{1}{2} \frac{1}{2} \frac{1}{2}$ positions.

\section{B. Linear displacement scattering}

Like short-range order scattering, linear displacement scattering is weak and can be parametrized by a few coefficients (the $\gamma_{l m n}^{x}$ are given in Table II). The linear displacement scattering of both planes shows a slight asymmetry across the Bragg reflections, best seen across the $0 \overline{2} 2$ reflection in the (111) surface and barely visible across $\overline{1} 11$ in the (110) surface. The asymmetry is smaller than in the scattering pattern from the bulk, also when using the speciesdependent static displacements of Ref. 1 together with the Hönl corrections for the present wavelength. The reason for this lies in the smaller magnitude of the nearest-neighbor displacement parameter.

\section{Configurational analysis}

Modelled crystals (of $32 \times 32 \times 32$ fcc unit cells with periodic boundary conditions) compatible with the set of Warren-Cowley short-range order parameters of Table I, were analyzed with respect to the distinguishable nearest-neighbor configurations, the 144 Clapp configurations. ${ }^{29}$ As for the case of the bulk microstructure (Table II of Steiner et al. ${ }^{1}$ ), the situation of $\mathrm{Pt}$ atoms around a $\mathrm{Rh}$ atom was considered. This choice was appropriate to distinguish the various super-

TABLE II. Fitted linear displacement parameters $\gamma_{l m n}^{x}$ for the Pt-47 at. \% Rh(110) and (111) surface at $1000 \mathrm{~K}$, and adjusted linear displacement parameters from the bulk (Ref. 1).

\begin{tabular}{cccc}
\hline \hline Imn & $(110)$ surface & $\begin{array}{c}\gamma_{l m n}^{x} \\
(111) \text { surface }\end{array}$ & Bulk Pt-Rh \\
\hline 110 & $-0.016(4)$ & $-0.004(2)$ & -0.0661 \\
200 & $-0.033(8)$ & $0.013(5)$ & -0.0201 \\
211 & $0.007(4)$ & $-0.016(3)$ & -0.0090 \\
121 & $0.001(2)$ & $-0.004(2)$ & -0.0038 \\
220 & $0.007(4)$ & & 0.0025 \\
\hline \hline
\end{tabular}



FIG. 4. Calculated short-range order scattering $I_{\text {SRO }}$ for a (001) plane, with the Warren-Cowley short-range order parameters from Table I of the (110) surface (upper triangle) and the (111) surface measurements (lower triangle). Data are given in 0.1 Laue units.

structures suggested by Lu et al. ${ }^{30}$ The configurations with the largest enhancement factors are summarized in Table III. The largest values of the enhancement factors are found for configuration $\mathrm{C} 134$. These values are much larger than those reported for bulk Pt-Rh (Ref. 1) or any "typical" short-range ordered binary alloy. This Clapp configuration C134 may be visualized as part of a stacking of three $\{111\}$ planes, with the central plane consisting exclusively of $\mathrm{Rh}$ atoms, while the adjacent $\{111\}$ planes have either two Pt atoms and one $\mathrm{Rh}$ atom, or one Pt atom and two Rh atoms. Such a sequence of a Pt-rich plane, a Rh-rich plane and another Pt-rich plane is reminiscent of the segregation profile found by Florencio et $a l .{ }^{5}$ for the three top layers of the $\{111\}$ surface of Pt- 44.8 at. $\%$ Rh and by Koller et al. ${ }^{10}$ for the $\{111\}$ microfacets of the reconstructed (110) surface of Pt-50 at. \% Rh. The abundance analysis thus indicates that the in-plane short-range order scattering, being the Fourier transformation of the three-dimensional Warren-Cowley short-range order parameters, also carries information on out-of-plane correlations.

TABLE III. Clapp configurations and site numbering [see Fig. 5 of Steiner et al. (Ref. 1)] of Pt atoms around a Rh atom with largest values of the enhancement factor.

\begin{tabular}{cccc}
\hline \hline \multirow{2}{*}{ Surface } & $\begin{array}{c}\text { Clapp configuration } \\
\text { of Rh atoms }\end{array}$ & Site occupancy & $\begin{array}{c}\text { Enhancement } \\
\text { factor }\end{array}$ \\
\hline \multirow{2}{*}{$(110)$} & C134 & $1-3,5,7-10,12$ & 120 \\
& C128 & $1-3,5,8,10-12$ & 2.8 \\
& C129 & $1-4,9-12$ & 2.5 \\
$(111)$ & C111 & $1-4,9,10,12$ & 2.1 \\
& C134 & $1-3,5,7-10,12$ & 228 \\
& C82 & $3,4,6,8,10,11$ & 3.4 \\
& C83 & $1,4,6,8,11,12$ & 2.4 \\
& C93 & $2,3,5,7-10$ & 2.1 \\
& C94 & $1-3,5,7,9,12$ & 2.1 \\
\hline \hline
\end{tabular}


For the characteristic configurations, next in importance in Table III, the enhancement factors are much lower and the configurations differ for both surfaces. For the (110) surface, the configurations next in importance are the building elements of superstructures where C128 is present in " 40 " and $\mathrm{C} 129$ in $\mathrm{L} 1_{0}$. Also, $\mathrm{C} 111$ is closely related to $\mathrm{L} 1_{0}$, with one wrong site occupancy. For the (111) surface, the subsequent Clapp configurations are still closely related with the leading configuration stacking sequence as for $\mathrm{C} 134$; they are either the building element of the $\mathrm{L}_{1}$ structure $(\mathrm{C} 82)$ or closely related to this structure (the configurations C83 and C94 only have one wrong site occupancy).

\section{DISCUSSION}

Diffuse x-ray scattering was separated into its contributions by least-squares fitting of the leading three-dimensional Fourier coefficients. This approach (in contrast to the twodimensional analysis done by Kimura et al. ${ }^{31}$ ) will allow out-of-plane scattering to be considered in further investigations. As the penetration depth of the $\mathrm{x}$-ray beam was about $2-3 \mathrm{~nm}, 8-12$ layers were illuminated.

The reason for assuming a cubic symmetry lies on the short segregation profile for the (110) and (111) surfaces; it only shows a large deviation from the average bulk concentration in the first two to three top layers of the crystal. With respect to the static displacements perpendicular to the surface, distortions down to the fifth layer were reported for the (110) surface and correlated with the missing-row reconstruction. ${ }^{10}$ Corresponding information for the (111) surface is missing.

In this investigation, however, no reconstruction was noted in the diffuse scattering of the (110) plane at elevated temperature. Note that at room temperature, the LEED pattern was consistent with a $(1 \times 3)$ reconstruction as in Ref. 10. There might be a change to an unreconstructed surface at elevated temperature; a transition from a $(1 \times 2)$ missing-row reconstruction of Pt-75 at. \% $\mathrm{Rh}(110)$ to an unreconstructed surface was reported for samples quenched from above $1020 \mathrm{~K}^{11}$

The fitted $B$ values of the total Debye-Waller factor differ considerably between both surfaces. Guided by the values of the main component at the top layer, platinum, one might expect (i) no dependence on the type of surface as the Debye temperatures of $\mathrm{Pt}(110)$ and (111) surfaces do not differ, (ii) a much larger value of $B$ than for the bulk as for Pt, $B$ is about $4.8 \times 10^{-2} \mathrm{~nm}^{2}$ (at $1000 \mathrm{~K}$ ) if one employs the Debye temperatures given by Lyon et al. ${ }^{32}$ Layer-dependent values of the Debye-Waller factor between $4.1 \times 10^{-2} \mathrm{~nm}^{2}$ (top layer) to $2.6 \times 10^{-2} \mathrm{~nm}^{2}$ (sixth layer) for the (110) surface were found by Robinson et al..$^{33}$ These large values of $B$ were attributed to the $(1 \times 3)$ missing-row reconstruction of pure $\mathrm{Pt}$ at room temperature. Less distortion may be expected in the present case of an unreconstructed surface as there is no reduced coordination related to the presence of the missing rows.

Correlations within the top layer as determined by Hebenstreit et al. ${ }^{10}$ by STM, are similar in sign and magnitude for the nearest neighbors, in spite of a different thermal history. The advantage of using grazing incidence diffuse scattering instead of STM with chemical contrast lies in the possibility to obtain a statistically relevant correlation function and to extend it to neighbors further away. The present investigations demonstrate that even small modulations can be analyzed applying a technique that has proved to be fruitful for the bulk microstructure. Additional out-of-plane scattering will stabilize the fitting algorithm and will provide detailed information on the microstructure beyond the global segregation profile.

\section{ACKNOWLEDGMENTS}

The authors thank E. Fischer for growing the single crystal used in the investigations. They very much appreciate the support of J. Gobrecht and St. Stutz in preparing the crystal surface and are grateful to the SLS team for assistance. This work was partially supported by the "Schweizerische Nationalfonds zur Förderung der wissenschaftlichen Forschung."
${ }^{1}$ Ch. Steiner, B. Schönfeld, M. J. Portmann, M. Kompatscher, G. Kostorz, A. Mazuelas, T. Metzger, J. Kohlbrecher, and B. Demé, Phys. Rev. B 71, 104204 (2005).

${ }^{2}$ T. B. Massalski, Binary Alloy Phase Diagrams (ASM International, Materials Park, OH, 1990).

${ }^{3}$ T. T. Tsong, D. M. Ren, and M. Ahmad, Phys. Rev. B 38, 7428 (1988).

${ }^{4}$ D. M. Ren, J. H. Qin, J. B. Wang, and T. T. Tsong, Phys. Rev. B 47, 3944 (1993).

${ }^{5}$ J. Florencio, D. M. Ren, and T. T. Tsong, Surf. Sci. 345, L29 (1996).

${ }^{6}$ B. Legrand and G. Tréglia, Surf. Sci. 236, 398 (1990).

${ }^{7}$ A. Drchal, A. Pasturel, R. Monnier, J. Kudrnovský, and P. Weinberger, Comput. Mater. Sci. 15, 144 (1999).

${ }^{8}$ A. V. Ruban and H. L. Skriver, Comput. Mater. Sci. 15, 119 (1999).

${ }^{9}$ E. Platzgummer, M. Sporn, R. Koller, S. Forsthuber, M. Schmid, W. Hofer, and P. Varga, Surf. Sci. 419, 236 (1999).
${ }^{10}$ R. Koller, Y. Gauthier, C. Klein, M. De Santis, M. Schmid, and P. Varga, Surf. Sci. 530, 121 (2003).

${ }^{11}$ E. L. D. Hebenstreit, W. Hebenstreit, M. Schmid, and P. Varga, Surf. Sci. 441, 441 (1999).

${ }^{12}$ E. Platzgummer, M. Sporn, R. Koller, M. Schmid, W. Hofer, and P. Varga, Surf. Sci. 423, 134 (1999).

${ }^{13} \mathrm{H}$. Dosch, Critical Phenomena at Surfaces and Interfaces (Springer, Berlin, 1992).

${ }^{14}$ M. Born and E. Wolf, Principles of Optics (Cambridge University Press, Cambridge, 1999).

${ }^{15}$ L. H. Schwartz and J. B. Cohen, Diffraction from Materials (Springer, Berlin, 1987).

${ }^{16}$ G. Kostorz, in Physical Metallurgy, edited by R. W. Cahn and P. Haasen (North-Holland, Amsterdam, 1996), p. 1115.

${ }^{17}$ B. Schönfeld, Prog. Mater. Sci. 44, 435 (1999).

${ }^{18}$ J. M. Cowley, J. Appl. Phys. 21, 24 (1950).

${ }^{19}$ B. Borie and C. J. Sparks, Acta Crystallogr., Sect. A: Cryst. Phys., Diffr., Theor. Gen. Crystallogr. 27, 198 (1971). 
${ }^{20}$ P. Georgopoulos and J. B. Cohen, J. Phys. Colloq. 38, C7-191 (1977).

${ }^{21}$ B. D. Patterson, R. Abela, H. Auderset, Q. Chen, F. Fauth, F. Gozzo, G. Ingold, H. Kühne, M. Lange, D. Maden, D. Meister, P. Pattison, Th. Schmidt, B. Schmitt, C. Schulze-Briese, M. Shi, M. Stampanoni, and P. R. Willmott, Nucl. Instrum. Methods Phys. Res. A 540, 42 (2005).

${ }^{22}$ S. Sasaki, KEK Report No. 88-14, Tsukuba, 1989.

${ }^{23}$ E. Vlieg, J. Appl. Crystallogr. 31, 198 (1998).

${ }^{24}$ G. H. Vineyard, Phys. Rev. B 26, 4146 (1982).

${ }^{25}$ B. E. Warren, X-ray Diffraction (Dover, New York, 1990).

${ }^{26}$ S. M. Collard and R. B. McLellan, Acta Metall. Mater. 40, 699
(1992).

${ }^{27}$ D. Maurer, R. Heichele, N. Lingg, V. Müller, and K. H. Rieder, J. Phys. IV C8, 535 (1996).

${ }^{28}$ D. T. Cromer, J. Chem. Phys. 50, 4857 (1969).

${ }^{29}$ P. C. Clapp, Phys. Rev. B 4, 255 (1971).

${ }^{30}$ Z. W. Lu, B. M. Klein, and A. Zunger, J. Phase Equilib. 16, 36 (1995).

${ }^{31}$ M. Kimura, J. B. Cohen, S. Chandavarkar, and K. Liang, J. Mater. Res. 12, 75 (1997).

${ }^{32}$ H. B. Lyon and G. A. Somorjai, J. Chem. Phys. 44, 3707 (1966).

${ }^{33}$ I. K. Robinson, P. J. Eng, C. Romainczyk, and K. Kern, Phys. Rev. B 47, 10700 (1993). 